\title{
Similar but not the same, the fission yeast processing bodies
}

\begin{abstract}
Processing bodies (P-bodies) are cytoplasmic RNA granules containing the Dcp1-Dcp2 decapping-enzymes where mRNA decay can occur. We have used the fission yeast Schizosaccharomyces pombe as a model system to study their structures and compositions. Because several components of P-bodies, such as the decapping enzyme and its co-activators, are found in both yeast and mammalian cells, it is believed that P-bodies are evolutionarily conserved among eukaryotes. However, we found that species diversity has developed; the protein compositions and underlying molecular mechanisms for their function can be distinct between species. These results led us to suggest that the whole complex including the decapping-enzyme and its co activators might have coevolved together and acquired additional proteins and different mechanisms for its function highlighting the importance of cross-species studies of this sort. Furthermore, we found that, although predominantly present in the cytoplasm, components of P-bodies also function in the nucleus. A function of Pdc2, the fission yeast ortholog of Pat1 protein, and the decapping enzyme Dcp1-Dcp2 function together with the 5'-3' exonuclease Dhp1 in the nucleus to regulate lncRNA by promoting its decapping/destruction was suggested.
\end{abstract}

Volume 3 Issue I - 2018

\author{
Shao Win Wang \\ Institute of Molecular \& Genomic Medicine
}

Correspondence: Shao Win Wang, National Health Research Institutes, 35 Keyan Road, Zhunan Town, Miaoli County 350, Taiwan, E-mail shaowinwang@nhri.org.tw

Received: November 21, 2017 | Published: January 17, 2018

Keywords: fission yeast, decapping, p-bodies

\section{Mini Review}

Cytoplasmic processing bodies (P-bodies) are RNA protein granules containing un translating mRNAs complexes with a set of translation repressors, the mRNA decapping machinery Dcp1-Dcp2 and the 5'-3'exonuclease Xrn1 where mRNA decay can occur and have merged as important sub cellular structures that are involved in mRNA metabolism. Although components of P-bodies have been described in Schizosaccharomyces pombe, a detailed description of P-bodies is still lacking. To this end, we have initiated a study to characterize the fission yeast $P$-bodies and several interesting aspects with regard to the protein composition between different species and underlying molecular mechanisms for their function were identified (Figure 1).1,2 Because of its importance, several model systems have been applied to study the structure of P-bodies, and studies in the budding yeast Saccharomyces cerevisiae have been crucial in unravelling P-body biology. However, there is a clear difference in terms of the decapping proteins presence in different organisms. In yeast, the catalytic subunit of decapping enzyme Dcp2 interacts directly with its cofactor Dcp $1 .{ }^{3}$ This complex has low intrinsic decapping activity and requires additional proteins including the enhancer of decapping 1-3 (Edc1-3), the heptametrical Lsm1-7 complex, the DExH/D-box RNA heli case 1 (Dhh1, RCK/p54 in mammals) and Pat1 for its full activity. All of these proteins concentrate in P-bodies and function differentially in activating decapping. ${ }^{4}$

In metazoans, Dcp1 and Dcp2 form direct but weak interactions that are facilitated by the decapping activator Edc4 (also known as Ge-1 or Hedls, Human enhancer of decapping large subunit). ${ }^{5}$ Edc4 4 is required for Dcp1-Dcp2 catalytic activity, ${ }^{6}$ which is not present in S. cerevisiae, indicating that species diversity has developed. The different protein composition of P-bodies between different species was highlighted by our recent study with the identification of the fission yeast Pdc1 (partner of decapping enzyme protein 1) as the functional homologue of Edc $4,{ }^{1}$ a third component of the decapping enzymes that is thought to be absent from fungi. Pdc1 forms a complex with Dcp2. Similar to Edc4; Pdc1 is an enhancer of decapping and plays a vital role in the formation of P-bodies. The protein composition and structure organization of fission yeast P-bodies is therefore more closely related to high eukaryote than that of S. Cerevisiae, making S. Pombe an ideal model for studying P-body biology. Intriguingly, our studies of characterization of Pdc2 (partner of decapping enzyme protein 2), the fission yeast ortholog of Pat1 protein complexing with Lsm17 to couple deadenylation and decapping, further reveal that even though species sharing similar protein composition, the underlying molecular mechanisms for their function can be distinct. ${ }^{10}$ Pat 1 was first identified as a protein associated with topoisomerase II. ${ }^{7}$ Since then, no other studies have focused on this nuclear function. Rather, Patl has been reported to play important roles in translational control and mRNA decay. ${ }^{8}$ It has been suggested that, in addition to facilitate decapping, S. Cerevisiae Pat 1 seems to play additional roles at earlier steps involving translational repression and mRNA rearrangement that set the stage for decapping. ${ }^{4}$ Translational repression activity has also been noted for Pat1 homolog's in Xenopus but in human. ${ }^{8,9}$ It is of interest to note that, as in the case of Pdc1, we found that the role of Pdc2 is similar to the Pat1 homolog in human but not in $S$. Cerevisiae, which appears to be involved only in mRNA degradation. Their interaction with Lsm1 differed significantly, too. These results led us to suggest that the whole complex including the decappingenzyme and its co activators might have coevolved together and acquired additional proteins and different mechanism for its function. In keeping with the role in mRNA decay, Pdc2 interacted with the decapping enzyme Dcp2 and its co-activators including Edc3, Pdc1 and Ste13, as well as the 5'-3' exoribonuclease Exo2, and localized to the P-bodies. P-body residency of Pat1 proteins also observed in other systems. However, it is not in complete agreement whether Pat1 proteins are essential for P-body assembly, possibly arising from different experimental or model systems. Our data showed that, similar to Pdc1, Pdc2 had a tendency to form aggregates that might facilitate P-body assembly. When over-expressed, Pdc2 affected the formation of P-bodies even in the absence of Pdc1, suggesting that although not 
absolutely essential for P-bodies assembly, Pdc2 also plays a role in P-body formation. Consistent with the proposed role of Pat1 proteins in coupling deadenylation and decapping, in addition to the interaction described above, Pdc2 interacted with Lsm1 and perhaps the whole Lsm1-7 complex. Although Pdc2 did not affect deadenylation, we found that Pdc2-Lsm1 complex was important for the recruitment of Ccr4 to P-bodies. Different from other components of P-bodies, we found that $\mathrm{Ccr} 4$ did not accumulate in fission yeast $\mathrm{P}$-bodies growing under favorable conditions and was only recruited to these foci upon stress in a manner that depended on the Pdc2-Lsm1 complex. Similarly, in S. Cerevisiae, only limited amount of Ccr4 were seen in P-bodies as compared with other factors during mid log growth, and were clearly observed to accumulate in P-bodies after deprivation of glucose. ${ }^{10}$ This is in contrast to the observations that human Pat $1 \mathrm{~b}$ tightly associated with the Ccr4-Caf1-Not1 deadenylation complex, ${ }^{9}$ and discrete Ccr4 foci can be readily detected in mammalian cells. ${ }^{11}$ In different species, the requirement of deadenylation in the assembly of P-bodies diverged, too. While deadenylases only have minor role in the assembly of P-bodies in yeast, ${ }^{10}$ deadenylation is a prerequisite for P-body formation in mammalian cells. ${ }^{12}$ Cross-species studies of this sort may provide useful information in understanding their evolutional path of these protein complexes.

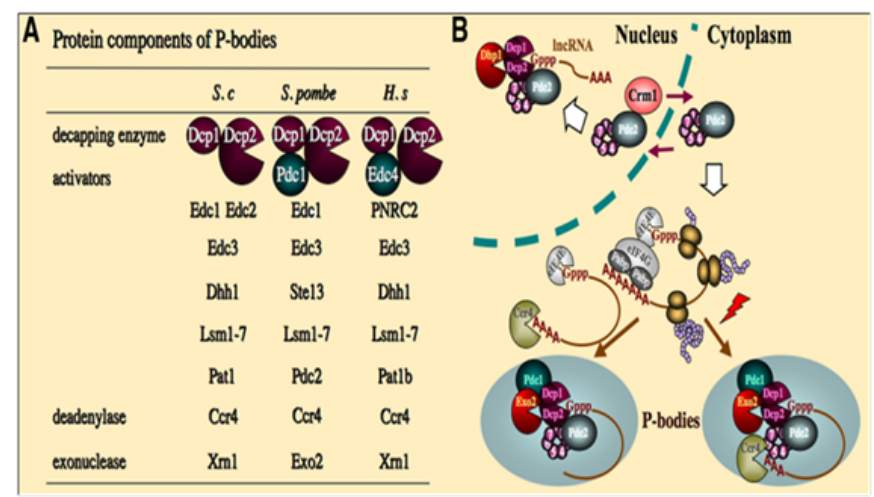

Figure I Schematic representation of (A) the protein components of P-bodies in different species and (B) model of their role in P-body function in S. Pombe [adapted from Wang et al., (2017) RNA 23:493-503].

In addition to its multiple regulatory roles in the cytoplasm, several lines of evidences suggested that Pat1 is a nucleo-cytoplasmic shuttling protein, ${ }^{10}$ and may have a nuclear role that remains to be clarified. In keeping with this notion, in the absence of Pdc2, Lsm1 was found to accumulate in the nucleus suggesting that Pdc2 shuttling between nucleus and cytoplasm plays a role in decreasing the nuclear concentration of Lsm1 to increase Lsm1 in the cytoplasm. In line with these results, we found that Pdc2 interacts with the nuclear 5'-3' exonuclease Dhp1, the ortholog of budding yeast Rat1 protein, and may function together with the decapping enzyme Dcp1-Dcp2 to regulate long non-coding RNA (lncRNA) in the nucleus. At present, the biological functions of the lncRNAs regulated by Pdc2 remain unclear as most of them located between previously uncharacterizedgenes that required further attention. Regardless of this, our study adds significantly to the versatility and complexity of the pathway and the mechanism in which decapping enzyme and its co-activators participated.

\section{Acknowledgements}

The work in author's lab was supported by the National Health Research Institute (MG-106-PP-08), and Ministry of Science and Technology (MOST106-2311-B-400-002), Taiwan.

\section{Conflict of interest}

Author declares that there is no conflict of interest.

\section{References}

1. Wang CY, WL Chen, Wang SW. Pdc1 functions in the assembly of P bodies in Schizosaccharomyces pombe. Mol Cell Biol. 2013;33(6):1244-1253.

2. Wang CY, YT Wang, WY Hsiao, et al. Involvement of fission yeast Pdc2 in RNA degradation and P-body function. RNA. 2017;23(4):493-503.

3. She M, CJ Decker, Chen N, et al. Crystal structure and functional analysis of Dcp2p from Schizosaccharomyces pombe. Nat Struct Mol Biol. 2006;13(1):63-70.

4. Nissan T, Rajyaguru P, She M, et al. Decapping activators in Saccharomyces cerevisiae act by multiple mechanisms. Mol Cell. 2010;39(5):773-783

5. Chang CT, N Bercovich, Loh B, et al. The activation of the decapping enzyme DCP2 by DCP1 occurs on the EDC4 scaffold and involves a conserved loop in DCP1. Nucleic Acids Res. 2014;42(8):5217-5233.

6. Fenger Gron M, Fillman C, Norrild B, et al. Multiple processing body factors and the ARE binding protein TTP activate mRNA decapping. Mol Cell. 2005;20(6):905-915.

7. Wang X, Watt PM, Louis EJ, et al. Pat1: a topoisomerase II-associated protein required for faithful chromosome transmission in Saccharomyces cerevisiae. Nucleic Acids Res. 1996;24(23):4791-4797.

8. Marnef A, Standart N. Pat1 proteins: a life in translation, translation repression and mRNA decay. Biochem Soc Trans. 2010;38(6):1602-1607.

9. Ozgur S, M Chekulaeva, Stoecklin G. Human Pat1b connects deadenylation with mRNA decapping and controls the assembly of processing bodies. Mol Cell Biol. 2010;30:4308-4323.

10. Teixeira D, Parker R. Analysis of P-body assembly in Saccharomyces cerevisiae. Mol Biol Cell. 2007;18(6):2274-2287.

11. Cougot N, Babajko S, Seraphin B. Cytoplasmic foci are sites of mRNA decay in human cells. J Cell Biol. 2004;165(1):31-40. 